\title{
Location-Triggered Code Execution - Dismissing Displays and Keypads for Mobile Interaction
}

\author{
W. Narzt and H. Schmitzberger \\ Johannes Kepler University Linz, Altenbergerstr. 69, A-4040 Linz, Austria \\ \{wolfgang.narzt, heinrich.schmitzberger\} @jku.at
}

\begin{abstract}
Spatially controlled electronic actions (e.g. opening gates, buying tickets, starting or stopping engines, etc.) require human attentiveness by conventional interaction metaphors via display and/or keystroke at the place of event. However, attentiveness for pressing a button or glimpsing at a display may occasionally be unavailable when the involved person must not be distracted from performing a task or is handicapped through wearable limitations (e.g. gloves, protective clothing) or disability. To automatically trigger those actions just at spatial proximity of a person, i.e. dismissing displays and keypads for launching the execution of electronic code in order to ease human computer interaction by innovative mobile computing paradigms is the main research focus of this paper.
\end{abstract}

Keywords: Location-Triggered Code Execution, Natural Interaction Paradigms.

\section{Introduction}

Currently available mobile location-based communication services enable their users to consume geographically bound information containing static text, images, sound or videos. Having arrived at previously prepared spots people are provided with information about the next gas station, hotels or sights of interest. More sophisticated variants of mobile location-based services also include dynamic links to locally available content providers. They additionally reveal the current gas price, vacancy status and reroute users to the online ticket service for tourists. Recently recognizable trends even consider individual user profiles as contextual constrains for supplying personalized information and as a technique to counteract spam and to selectively address content to specific user groups.

However, the potentials of mobile interaction are far beyond being exploited, considering limiting factors preventing users from attending to the information screen of their mobile device e.g. while driving in a car. Active interaction may also be hindered when people are handicapped or requested to wear gloves, safety glasses or protective suits in order to perform a working task. What is the use of perfectly filtered, personalized dynamic information when the addressee is not able to perceive or react to it? We expect mobile services to support the users in their tasks by automatically triggering (personally authorized) electronic actions just at spatial proximity of approaching users without the needs of glimpsing at displays, typing, clicking or pressing buttons. 
As a consequence, mobile location-based services are not solely regarded as information providers but also as action performers. The context location in combination with personalized access privileges and further quantifiably sensory input are the triggers for opening gates, automatically stopping engines in danger zones or validating tickets at entrance areas. Hence, people are able to continue their natural behavior without being distracted from their focused task and simultaneously execute an (assumed incidental but necessary) action. The users' mobile devices enabling locationtriggered code execution remain in their pockets.

\section{Related Work}

In this paper, we are primarily concerned with intuitive human computer interaction in mobile computing scenarios derived from the spatial context of the respective user. In this regard, the notion "context" has been issued in numerous publications and is widely thought of as the key to a novel interaction paradigm. In [1] Dourish analyzed the role context plays in computer systems and claimed that future computing scenarios will move away from the traditional single user desktop applications employing mouse, keyboard and computer screen. In [2] the usage of context in mobile interactive systems has been analyzed. Dix et al. determined the relevance of space and location as fundamental basis for mobile interaction. [3] studies the user's needs for location-aware mobile services. The results of the conducted user evaluation highlight the need for comprehensive services as well as for seamless service chains serving throughout a user's mobile activity.

To achieve broad user acceptance mobile computing research is confronted with the issue of seamless transitions between the real and the digital world [4] without distracting the user's attention [5]. Modern solution approaches use Near Field Communication (NFC) for contactless initiated actions following the same objectives of dismissing the conventional display and key-controlled interaction paradigm in order to claim a minimum of attention for performing an action at a place of event (e.g. SkiData - contactless access control in skiing areas through RFID). However, the disadvantage in this solution lies within the fact that every location which is supposed to trigger an electronic action has to consider mandatory structural measures for engaging the NFC principle. Beyond, a remaining part of attention is still required as users are supposed to know the position of the NFC system and bring up the RFID tag or reader (depending on which part of the components carries the reading unit) close to the system for proper detection. Regarding the structural measures for implementing NFC, this technology is only marginally applicable causing financial and environmental impairments.

In earlier research on context with respect to HCI the notion implicit interaction appears [6]. Implicit interaction denotes that the application will adapt to implicitly introduced contextual information learned from perceiving and interpreting the current situation a user is in (i.e. the user's location, surrounding environment, etc.). In [7] this definition has been refined and split up separating between implicit input and implicit output. Implicit input is regarded as action performed by a human to achieve a specific goal while secondary captured and interpreted by a system. Implicit output is described as not directly related to an explicit input but seamlessly integrated with 
the environment and the task of the user. The approach presented in this paper proposes a means of combining implicit input and output to achieve a minimum of user distraction.

Up to now, HCI research already offers numerous contributions on implicit interaction from an application point of view. In [8] the usage of accelerometer data attached to digital artifacts has been exploited to implicitly grant access to a room. The Range Whiteboard [9] explores implicit interaction on public displays supporting co-located, ad-hoc meetings. Similar to this work interactive public ambient displays are explored towards interaction in [10]. Here, the contextual focus lies on body orientation and position cues to determine interaction modes. In [11] the personalization of GIS area maps is realized by monitoring users' implicit interaction with maps. All these works on implicit interaction strongly focus on the primary role of the user in the interaction process and the modalities of interacting with ubiquitous computers respectively digital artifacts.

Spontaneous interaction triggered upon physical proximity was further studied in numerous works [12] [13] [14]. These approaches share the aspect that radio sensors are used to determine mutual proximity between smart artifacts and humans. The simplest form of smart artifacts are Smart-Its [15], small computing devices that can be attached unobtrusively to arbitrary physical objects in order to empower these with processing, context-awareness and communication. Smart-Its are designed for ad hoc data exchange among themselves in spatial proximity. In [16] Gellersen et al. underlined the importance of awareness of the environment and of the situation for inferring behavior of mobile entities.

Many researchers have focused on identifying smartness in mobile systems. As for the reason of usability this paper focuses on implementing smart environments rather than smart tools or appliances. Key issues of such smart environments have already been discussed recently. The ReMMoC system [17], a web service based middleware solution, deals with the problem of heterogeneous discovery and interaction protocols encountered in mobile applications. In [18] interaction with physical objects is supported by a web service backend system providing mobile phone users with access to associated services. Common to most solutions for mobile interaction is the usage of spatial context. Zambonelli et al. presented the spatial computing stack [19], a framework to overcome the inadequacy of traditional paradigms and to accentuate the primary role of space in modern distributed computing. Generally, it represents virtual environments and their physical counterpart as a common information space for creating awareness among the participants.

\section{Architecture}

Similar to mobile telecommunication services we propose a distributed provider model as the basis for realizing a common information space enabling worldwide unbounded mobile location-based communication services. This proven model allows users to join the provider of their choice and guarantees scalability of the service as each provider only handles a limited number of clients [4]. Every provider stores a set of geographically linked information in appropriate fast traversable geo-data structures (e.g. r-trees) containing hierarchically combinable content modules (which we 
call gadgets) for text, pictures videos, sound, etc. The name gadget already refers to a possible activity within a module and is the key for a generic approach of integrating arbitrary electronic actions to be triggered automatically on arriving users. The main focus of designing an architecture for location-triggered code execution is high extensibility to third-party systems, for the number and variety of non-recurring electronic possibilities is unforeseeable and simultaneously enriches the potentials of such a service. Fig. 1 illustrates the common principles of a flexible component architecture which enables fast connections to third party systems:

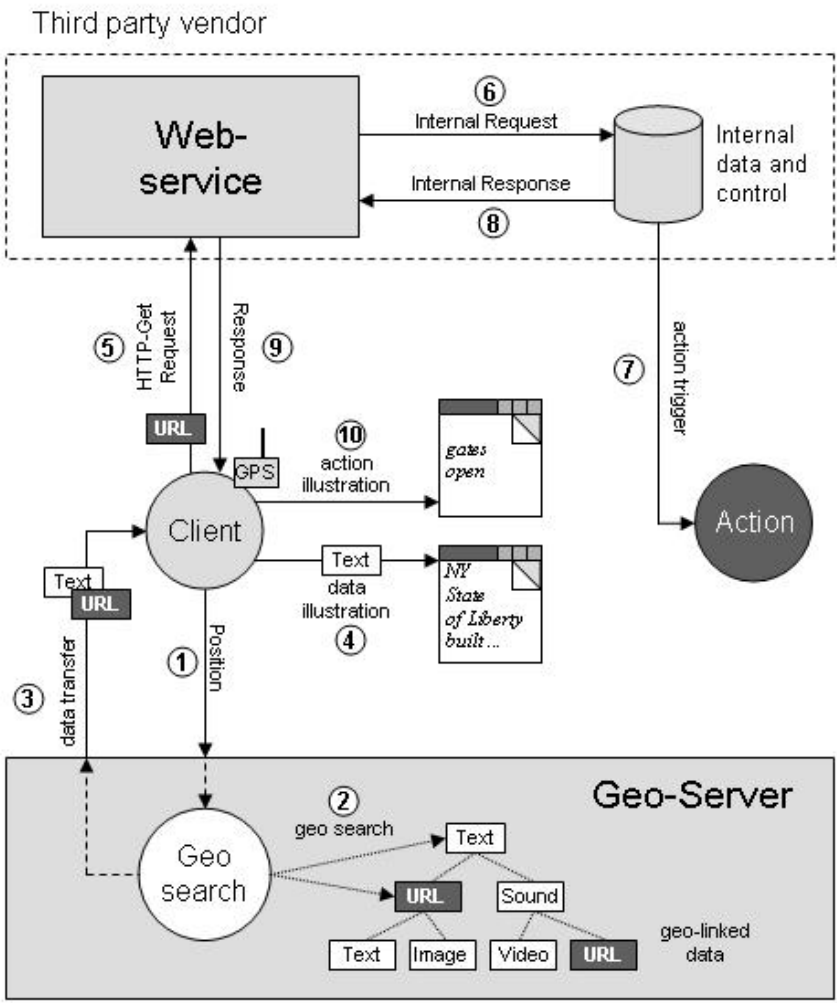

Fig. 1. Extensible Component Architecture for Location-Triggered Code Execution

The basic technical approach is a client-server model where clients repetitively transmit their own (commonly by GPS-based) position to a server (1) which evaluates the geo-data considering visibility radiuses and access constraints (2) and transmits the corresponding results back to the clients (3). Generally, when the transmitted information contains conventional gadgets as text and pictures, it is immediately displayed on the output device of the client (4). The basic idea for executing code is to use the gadget metaphor and store executable code inside instead of text or binary picture data (smart gadgets). Whether this piece of code is executed on the client or the server is primarily irrelevant for the paradigm of automatically triggering actions at certain locations. 
However, where to execute the code is crucial for system compatibility and extensibility issues. The client as the executing platform brings up portability tasks at every new third-party connection, as there is possibly more than one system implementation for covering multiple mobile platforms. The server on the other hand would need an elaborate plug-in mechanism for covering new third-party connections and is then still faced with the problem of integrating code from varying third-party operating systems.

In order to solve this conflict, we propose a web-service-based mechanism which is both effective and simple to extend: Smart gadgets do not actually contain executable code but a simple URL to a remote web-service which is the actual component to execute the code. Every third-party vendor provides a web-service and decides about the URL and its parameters on her own. When a client receives information containing a smart gadget, its URL is resolved in some kind of HTTP-request (5) which is handled internally (6) and finally triggers the desired electronic action (7). A response back to the client (8)(9) can additionally be illustrated as a visual confirmation of the thirdparty system whether the action could have been executed successfully or not (10).

This architecture comprises several advantages:

- In order to execute location-based code clients just have to handle standardized HTTP-requests. A majority of currently utilized mobile platforms support these mechanisms.

- The system does not run into compatibility or portability problems, for the executable code is exclusively run on the platform of the corresponding web-service.

- Commonly, location-triggered actions are provided by third-party vendors (e.g. opening of garage doors, gates, starting or stopping of machines, etc.). Using a web-based approach, external systems can easily be linked without compiling the core system or adding plug-ins to it.

- Most important for third-party vendors: Their internal data representations, servers and control units are hidden from the publically accessible location-based service, guaranteeing a maximum of data security for the vendors.

Summarizing all those architectural thoughts, location-triggered code execution is easily achievable by using conventional (GPS- and wireless-enabled) devices and services and adding web-services via a smart gadget mechanism to them. Simple standardized HTTP-requests enable arbitrary integration of third-party systems without structural measures as they are e.g., mandatory for NFC systems.

\section{Implementation}

In the course of a research project, the Johannes Kepler University of Linz, Siemens Corporate Technology in Munich and the Ars Electronica Futurelab in Linz have developed a novel location-based information and communication service for mobile devices facing the challenges of natural interaction triggered by geographical closeness without display and keypad. It enables users to arbitrarily post and consume information in real locations for asynchronous one-to-many or one-to-any communication having time-driven and contextual perceptibility; and it provides a framework interface for extending the functional range of the service, especially for adding new smart elements by third party vendors. 

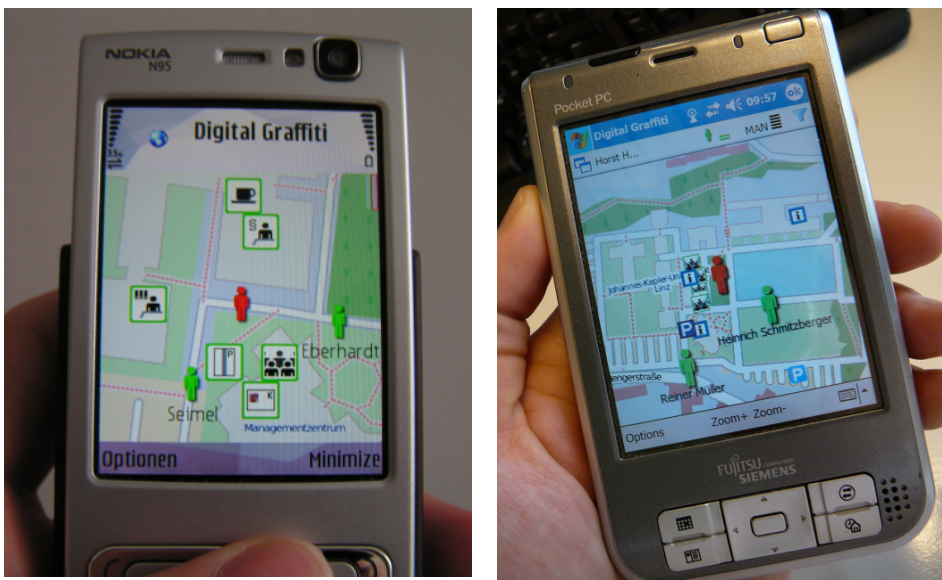

Fig. 2. Location-Based Client for Cell Phones and PDAs

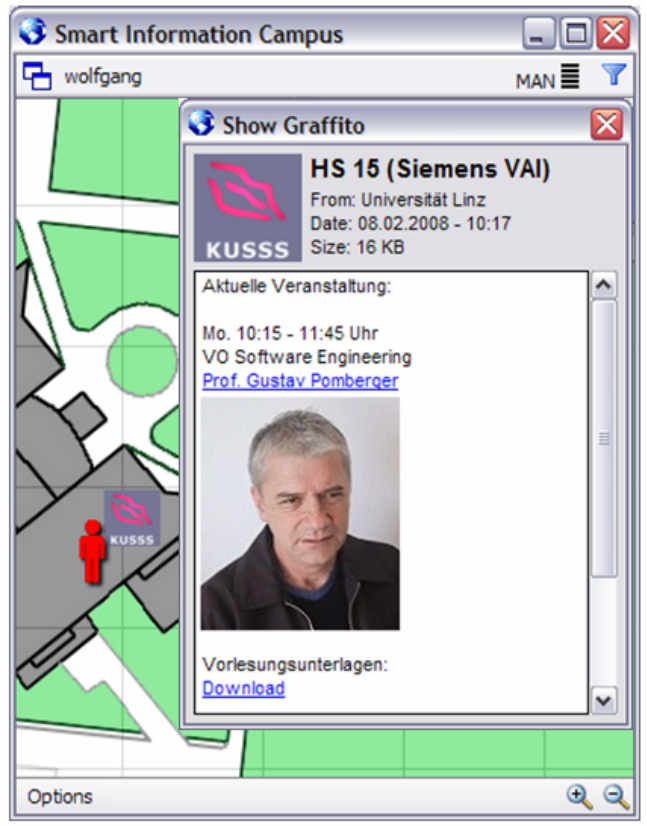

Fig. 3. Up-to-date Lecture Information at the campus of the University of Linz

The server component of our proposed architecture (as sketched in Fig. 1) has been implemented as a multithreaded $\mathrm{C}++$ application capable of providing different kinds of gadgets regarding the user's current position. Localization is selectively accomplished via GPS, WLAN triangulation, RFID- or Bluetooth-based positioning.

In order to guarantee multi-platform compatibility, the client component uses a slim J2ME system core supporting various mobile platforms including cell phones, PDAs and conventional notebooks. Data transmission is implemented for the most 
common wireless communication techniques (GPRS, UMTS, WLAN, Bluetooth). Fig. 2 shows snapshots of the client software for mobile phones (left) and PDAs (right).

The web-based third-party component can be deployed to external server systems and uses REST technology (Representational State Transfer, a client-server communication architecture) to offer access to and control of its internal set of functions. In order to prevent abuse of the service the component includes identification and authorization mechanisms, only granting access to registered users.

The applicability of this framework architecture is currently being demonstrated in the course of a first reference implementation at the campus of the University of Linz, available for students, academics and maintenance staff. At the moment, the campuswide deployment of third-party components is restricted to solely embedding dynamic content from several university-related information systems (e.g. event management, study support system, lecture room occupation plan). However, the mechanism already follows the principle of location-triggered code execution as proposed in chapter 3. For instance, students are able to obtain up-to-date lecture information at special proximity to respective auditorium halls (see Fig. 3).

\section{Fields of Application}

The project described in the previous chapter has already attracted potential customers from industry and the consumer market, who have already expressed their interest in adopting our service in their business fields. Due to the manifold fields of interested industry segments, we could identify four different types of relevant locationtriggered actions:

1. Actions that should be executed when users approach at a geographical point.

2. The execution is due to entering an area (e.g. a room)

3. Also the opposite is valid for location-triggered actions (e.g. leaving a room)

4. For certain places, users are supposed to reside for a predefined period of time before actions are executed.

All those examples can additionally be enriched by considering the current heading of a user, i.e., from which direction is the user approaching a point or entering or leaving an area? The following use-cases demonstrate examples of (already implemented and planned) location-triggered actions validating the functional scope and the extensibility of the system:

To start with, a common area of application for triggering actions at points of arrival is derived from logistics requirements: Carriers arriving at their designated destination automatically engage the process of loading or unloading cargo controlling e.g., local conveyor belts, and affecting storage management software for altering working procedures.

Another use-case for location-triggered actions on entering an area has already resulted in a real business scenario: A producer of golf carts is equipping his vehicles with a location-based information system displaying overviews on the players' current position at the golf course revealing distances to holes and obstacles. When players 


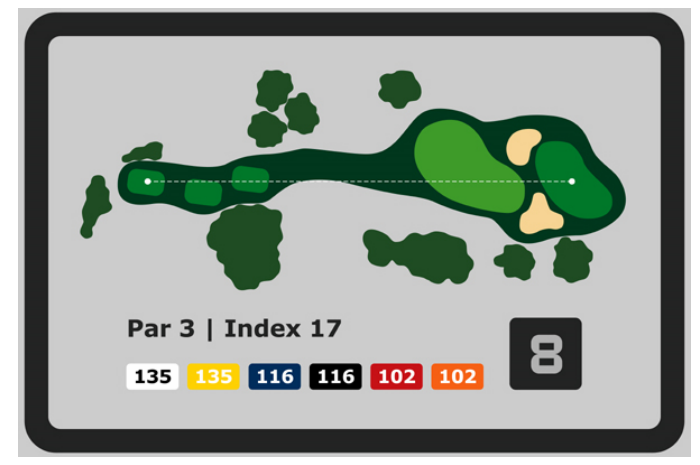

Fig. 4. Alerting or stopping Engines when driving on Fairways or Greens

try to drive on forbidden fairways and greens (marked in light green in Fig. 4) the system automatically alerts to the operator or even automatically stops engines.

Leaving an area may be interesting for power consumption issues. A household being aware of two persons living in it and recognizing the dwellers' positions leaving a selected region around the property triggers electronically controllable units (e.g. lights, central heating, door lock, etc.) to be switched off or to be locked in order to decrease power consumption. In contrast to existing smart power consumption solutions, location-triggered code execution does not need any additional sensory gadgets for context recognition and an electronic backbone to keep them working [20]. The personal mobile device which is powered on anyway is the only gadget required to be turned on for enabling power consumption.

Concerning the application field of industrial security mechanisms where people must leave contaminated zones after strict time slots, the fourth type of triggering actions can be applied: The maintenance staff is restrained by protective clothing needs to focus on their primary repairing tasks and is unable to monitor security displays. The alarm automatically signalizes upcoming hazardous situations to each worker individually and to the supervising operators.

\section{Conclusion and Future Work}

Location-triggered code execution enables a variety of innovative interaction mechanisms, neither distracting the users' attention from their currently performed tasks nor requiring structural measures for implementing it. For the initiation of electronically controlled actions users can abstain from conventional interaction techniques using displays and keystrokes. Solely, their physical presence is the trigger for real events. Users are simply requested to carry the enabling infrastructure, i.e., a mobile, wireless communication device equipped with some kind of tracking technology in their pockets. Currently available cellular phones and PDAs already fulfill these technical requirements and are suited as client devices for instantiating location-triggered code execution. Generic extension to external systems via web-services is the key for implementing a manifold of application scenarios by third-party vendors. Without interfering into the core system, new electronic functions can be adopted by using simple, standardized methods widening the palette of applications unboundedly. 
Our prototype at the University of Linz already shows the applicability of the proposed concept focusing on eliminating key strokes for mobile computing interaction. However, some interaction modalities still depend on the use of arbitrary forms of display metaphors (visual, acoustic, haptic), yet. Future work will comprise further studies on coupling location context with triggering actions in order meet the paradigm of display- and keyless mobile interaction.

\section{References}

1. Dourish, P.: What we talk about when we talk about context. Personal Ubiquitous Computing 8(1), 19-30 (2004)

2. Dix, A., Rodden, T., Davies, N., Trevor, J., Friday, A., Palfreyman, K.: Exploiting space and location as a design framework for interactive mobile systems. ACM Trans. Comput.Hum. Interact. 7(3), 285-321 (2000)

3. Kaasinen, E.: User needs for location-aware mobile services. Personal Ubiquitous Comput. 7(1), 70-79 (2003)

4. Narzt, W., Pomberger, G., Ferscha, A., Kolb, D., Müller, R., Wieghardt, J., Hörtner, H., Haring, R., Lindinger, C.: Addressing concepts for mobile location-based information services. In: Proceedings of the 12th International Conference on Human Computer Interaction HCI 2007 (2007)

5. Ishii, H., Ullmer, B.: Tangible bits: towards seamless interfaces between people, bits and atoms. In: CHI 1997: Proceedings of the SIGCHI conference on Human factors in computing systems, pp. 234-241. ACM, New York (1997)

6. Schmidt, A.: Implicit human computer interaction through context. Personal Technologies (2000)

7. Schmidt, A., Kranz, M., Holleis, P.: Interacting with the ubiquitous computer: towards embedding interaction. In: sOc-EUSAI 2005: Proceedings of the 2005 joint conference on Smart objects and ambient intelligence, pp. 147-152. ACM, New York (2005)

8. Antifakos, S., Schiele, B., Holmquist, L.E.: Grouping mechanisms for smart objects based on implicit interaction and context proximity. Interactive Posters at UbiComp 2003 (2003)

9. Ju, W., Lee, B.A., Klemmer, S.R.: Range: Exploring implicit interaction through electronic whiteboard design. Technical report, Stanford University, HCI Group (2006)

10. Vogel, D., Balakrishnan, R.: Interactive public ambient displays: transitioning from implicit to explicit, public to personal, interaction with multiple users. In: UIST 2004: Proceedings of the 17th annual ACM symposium on User interface software and technology, pp. 137-146. ACM Press, New York (2004)

11. Weakliam, J., Bertolotto, M., Wilson, D.: Implicit interaction profiling for recommending spatial content. In: GIS 2005: Proceedings of the 13th annual ACM international workshop on Geographic information systems, pp. 285-294. ACM, New York (2005)

12. Ferscha, A., Mayrhofer, R., Oberhauser, R., dos Santos Rocha, M., Franz, M., Hechinger, M.: Digital aura. In: Advances in Pervasive Computing. A Collection of Contributions Presented at the 2nd International Conference on Pervasive Computing (Pervasive 2004), Austrian Computer Society (OCG), Vienna, Austria, April 2004, vol. 176, pp. 405-410 (2004)

13. Kortuem, G., Schneider, J., Preuitt, D., Thompson, T.G.C., Fickas, S., Segall, Z.: When peer-to-peer comes face-to-face: Collaborative peer-to-peer computing in mobile ad hoc networks. In: Proceedings of the First International Conference on Peer-to-Peer Computing, P2P 2001 (2001) 
14. Brunette, W., Hartung, C., Nordstrom, B., Borriello, G.: Proximity interactions between wireless sensors and their application. In: WSNA 2003: Proceedings of the 2nd ACM international conference on Wireless sensor networks and applications, pp. 30-37. ACM Press, New York (2003)

15. Gellersen, H., Kortuem, G., Schmidt, A., Beigl, M.: Physical prototyping with smart-its. IEEE Pervasive Computing 3(3), 74-82 (2004)

16. Gellersen, H.W., Schmidt, A., Beigl, M.: Multi-sensor context-awareness in mobile devices and smart artifacts. Mob. Netw. Appl. 7(5), 341-351 (2002)

17. Grace, P., Blair, G.S., Samuel, S.: A reflective framework for discovery and interaction in heterogeneous mobile environments. SIGMOBILE Mob. Comput. Commun. Rev. 9(1), 2-14 (2005)

18. Broll, G., Siorpaes, S., Rukzio, E., Paolucci, M., Hamard, J., Wagner, M., Schmidt, A.: Supporting mobile service usage through physical mobile interaction. In: Proceedings of the Fifth IEEE international Conference on Pervasive Computing and Communications, PERCOM (2007)

19. Zambonelli, F., Mamei, M.: Spatial computing: an emerging paradigm for autonomic computing and communication. In: 1st International Workshop on Autonomic Communication, Berlin (October 2004)

20. Ferscha, A., Emsenhuber, B., Gusenbauer, S., Wally, B.: PowerSaver: Pocket-Worn Activity Tracker for Energy Management. In: Adjunct Proceedings of the 9th International Conference on Ubiquitous Computing UbiComp 2007, pp. 321-324 (2007) 